Research Article

\title{
Comparison of Essential Oils of Houttuynia cordata Thunb. from Different Processing Methods and Harvest Seasons Based on GC- MS and Chemometric Analysis
}

\author{
Xue Pan $\left(\mathbb{D},{ }^{1,2,3}\right.$ Haiying Li ${ }^{D},{ }^{1,2,3}$ Dingfang Chen, ${ }^{1}$ Jinjin Zheng, ${ }^{1}$ Longhua Yin, ${ }^{1}$ Juan Zou, ${ }^{1}$ \\ Yalun Zhang, ${ }^{1}$ Kaiwen Deng, ${ }^{4}$ Meifeng Xiao, ${ }^{1,2,3}$ Lei Meng $\mathbb{1},{ }^{1,2,3}$ and Fuyuan He $\mathbb{\circledR}^{1,2,3}$ \\ ${ }^{1}$ College of Pharmacy, Hunan University of Chinese Medicine, Changsha 410208, China \\ ${ }^{2}$ Hunan Provincial Key Laboratory of Drugability and Preparation Modification of TCM, Changsha 410208, China \\ ${ }^{3}$ Supramolecular Mechanism and Mathematic-Physics Characterization for Chinese Materia Medica, \\ Hunan University of Chinese Medicine, Changsha 410208, China \\ ${ }^{4}$ The First Affinity Hospital, Hunan University of Chinese Medicine, Changsha 410007, China
}

Correspondence should be addressed to Lei Meng; 27783513@qq.com and Fuyuan He; pharmsharking@163.com

Xue Pan and Haiying Li contributed equally to this work.

Received 9 April 2021; Revised 29 June 2021; Accepted 5 July 2021; Published 17 July 2021

Academic Editor: Pavel Nesterenko

Copyright $\odot 2021$ Xue Pan et al. This is an open access article distributed under the Creative Commons Attribution License, which permits unrestricted use, distribution, and reproduction in any medium, provided the original work is properly cited.

\begin{abstract}
Houttuyniae Herba (HH) refers to the dried aerial part of Houttuynia cordata Thunb. (DHC) or the fresh whole grass of Houttuynia cordata Thunb. (FHC), where DHC are harvested in summer and FHC around the year. However, harvest seasons and processing methods (i.e., medicinal parts and drying process) might affect the quality of $\mathrm{HH}$. To compare the essential oils (EOs) of DHC and FHC and their two harvest seasons, GC-MS analysis combined with chemometric analysis was applied. The results showed that the oil yield of FHC $(0.076 \pm 0.030 \%)$ was higher than that of DHC $(0.038 \pm 0.029 \%)$, and oil yield was higher in summer than in autumn $(0.044 \pm 0.029 \%$ for DHC1, $0.036 \pm 0.028 \%$ for DHC2, $0.084 \pm 0.026 \%$ for FHC1, and $0.067 \pm 0.033 \%$ for FHC2, respectively). Moreover, hierarchical cluster analysis (HCA) and principal component analysis (PCA) successfully distinguished the chemical constituents of DHC and FHC oils. Additionally, according to orthogonal partial least squares discriminant analysis (OPLS-DA), eleven components were selected as chemical markers for discriminating DHC and FHC, and two and four chemical markers for discriminating two harvest seasons of DHC and FHC, respectively. Among these markers, the average contents of $\alpha$-pinene, limonene, $\beta$-phellandrene, $\alpha$-terpineol, 4 -tridecanone, and ethyl decanoate were higher in FHC oils. In contrast, the average contents of nonanal, 1-nonanol, $\beta$-cyclocitral, $n$-hexadecanoic acid, and octadecanol were higher in DHC oils. Additionally, the contents of 4tridecanone and ethyl decanoate were both higher in DHC1 oils than in DHC2 oils. Moreover, the contents of $\beta$-myrcene and $\beta$-phellandrene were higher in FHC1 oils, while the contents of 2,6-octadien-1-ol, 3,7-dimethyl-, acetate, and (z)-phytol were higher in FHC2 oils. For these reasons, this study provides a scientific basis for quality control and clinical medication.
\end{abstract}

\section{Introduction}

Houttuynia cordata Thunb. (HC), a promising herbal medicine of the family Saururaceae, is mainly distributed in Asia, including China, India, Thailand, Japan, and Korea. It has a long history of clinical use in traditional Chinese medicine for its several functions, including antifebrile and detoxification, eliminating carbuncle and discharging pus, and promoting diuresis and relieving stranguria [1]. HC mainly contains essential oil (EO), organic acids, flavonoids, phenols, polysaccharides, and alkaloids. EO is one of the main effective parts of $\mathrm{HC}$ because it has a variety of pharmacological activities, such as antibacterial, anti-inflammatory, anticancer, antifungal, antiviral, antihistamine, and antidiabetic activities [1-3]. Thus, investigating the chemical compositions of EO could be an effective approach to evaluate the quality of HC. To date, many researchers studied the chemical constituents of EO of HC. Some 
research teams revealed the main effective components of EO of HC were 2-undecanone, decanal, decanoyl acetaldehyde, $\alpha$-pinene, camphene, $\beta$-pinene, $\beta$-myrcene, lauraldehyde, bornyl acetate, limonene, caryophyllene, 4terpineol, nonanol, and caryophyllene oxide [3]. In addition, some investigators suggested that medicinal parts, production areas, harvest seasons, and processing methods might influence the chemical composition, which in turn affect the therapeutic efficacy of the herbs [1,3-5].

According to the 2020 edition of Chinese pharmacopoeia, DHC and FHC are indiscriminately used as one herb medicine called Yuxingcao in Chinese (Houttuyniae Herba), and DHC are harvested in summer and FHC around the year. The different processing methods (i.e., medicinal parts and drying process) and harvest seasons might influence their chemical composition and quality. As DHC and FHC are indiscriminately used in clinic and EO is regarded as one of the main effective parts [1], it is necessary to compare the EOs of $\mathrm{DHC}$ and $\mathrm{FHC}$ and their two harvest seasons systematically and comprehensively. GC-MS fingerprinting technology is the routine method to analyze EO compounds of HC. However, according to our knowledge, only one study directly compared the content differences of 16 components. The authors found 5 components with obvious differences of 2 batches of DHC and 8 batches of FHC from Emei in China [1]. This intuitive comparison could find some components with obvious differences. However, conventional mutual chemical comparison cannot determine elements that result in quality variance. In recent years, chemical fingerprints combined with chemometric analysis such as HCA, PCA, and OPLS-DA have become powerful tools for the identification and quality assessment of traditional Chinese medicine [6-8].

In this study, GC-MS analysis combined with chemometric methods was first applied to compare EOs of DHC and FHC and their two harvest seasons systematically. HCA and PCA were utilized for the classification and distinction of their EOs. Then, OPLS-DA was further employed to identify the potential chemical markers responsible for the discrimination. In addition, the relative content difference of these chemical markers in DHC and FHC and their two harvest seasons were analyzed, and their pharmacological properties were considered. The aim of this study is to investigate the differences of EOs of DHC and FHC and their two harvest seasons and to provide scientific basis for quality control and clinical medication.

\section{Materials and Methods}

2.1. Plant Material and Isolation of Essential Oils. Twenty-one batches of DHC and twenty-three batches of FHC were collected from the main production areas in China such as Zhejiang, Guangxi, Jiangxi, Hunan, Guizhou, Sichuan, and Jiangsu, at two harvest seasons (summer season and autumn season). DHC were obtained from the aerial parts of fresh $\mathrm{HC}$, which were dried naturally until the weight was unchanged, while FHC were obtained from the whole grass of fresh HC. The plants were identified by Professor Xiaojiang Zhou (School of pharmacy, Hunan
University of Chinese Medicine, Changsha, China). Among all the samples, DHC were labeled as DHC (batches in summer labeled DHC1-1 to DHC1-11 and batches in autumn labeled DHC2-1 to DHC2-10), while FHC were labeled as FHC (batches in summer labeled FHC1-1 to FHC112 and batches in autumn labeled FHC2-1 to FHC2-11).

DHC (120 g) or FHC (600 g) were cut into small segments $(2-3 \mathrm{~cm})$ and subjected to hydrodistillation in a Clevenger-type apparatus for four hours for isolation of EOs. The oil yields (\%, v/w) were calculated in milliliters of oil per $100 \mathrm{~g}$ of FHC and the aerial parts of fresh HC for preparing DHC. After being dehydrated over anhydrous sodium sulphate, it was stored at approximately $4^{\circ} \mathrm{C}$, ready for GCMS detection. Each $\mathrm{EO}$ was diluted 20 times with n-hexane before GC-MS analysis.

2.2. GC-MS Analysis. GC-MS analysis was performed on GC-MS-QP2010 (Shimadzu, Japan) equipped with the DB-5 capillary column $(60 \mathrm{~m} \times 250 \mu \mathrm{m} \times 0.25 \mu \mathrm{m}$ film thickness; Agilent). A volume of $1 \mu \mathrm{L}$ of each sample was injected. The carrier gas was $\mathrm{He}$ (purity $>99.999 \%$ ) at $1.5 \mathrm{~mL} / \mathrm{min}$ with a split ratio of $10: 1$ and the injector temperature was $250^{\circ} \mathrm{C}$. The oven temperature was set at $80^{\circ} \mathrm{C}$ for 2 minutes and then increased to $125^{\circ} \mathrm{C}$ at a rate of $10^{\circ} \mathrm{C} / \mathrm{min}$ and maintained for $5 \mathrm{~min}$, to $165^{\circ} \mathrm{C}$ at a rate of $10^{\circ} \mathrm{C} / \mathrm{min}$ and held for $10 \mathrm{~min}$, to $185^{\circ} \mathrm{C}$ at a rate of $2^{\circ} \mathrm{C} / \mathrm{min}$, and finally to $240^{\circ} \mathrm{C}$ at a rate of $10^{\circ} \mathrm{C} / \mathrm{min}$ and lasted for $20 \mathrm{~min}$. The mass spectrometer was operated in electron impact (EI) mode at $70 \mathrm{eV}$ in a scan range of $35-500 \mathrm{~m} / \mathrm{z}$. The temperature of the ion source and transmission line was $250^{\circ} \mathrm{C}$ and $280^{\circ} \mathrm{C}$, respectively. The solvent delay was $4 \mathrm{~min}$.

2.3. Data Preprocessing. Peaks were picked up with the signal-to-noise ratio $(\mathrm{S} / \mathrm{N})>6$. Raw data of GC-MS were transformed into MZXML format by the Shimadzu postrun workstation and finally transformed into ABF format and then processed by Mass Spectrometry-Data Independent Analysis (MS-DIAL) software to detect volatile compounds features and align all the peaks. The components of EO were identified and screened by the MS-DIAL and National Institute of Standards and Technology (NIST) 17.0 Mass Spectra Database. The semiquantitative analysis of volatile compounds was performed by comparing their peak areas in the GC-MS total ion chromatogram. The percentage compositions of common compounds were calculated by the area normalization method.

2.4. Statistical Analysis. Heatmap and HCA were generated on the MetaboAnalyst5.0 (https://www.metaboanalyst.ca/) to display the overall difference of chemical compositions of DHC and FHC. PCA and OPLS-DA were applied to investigate the distinction of chemical compositions of DHC and FHC by SIMCA-P 15.0 (Umetrics AB, Umea, Sweden). The significant chemical markers were evaluated based on their $p$ value and differing variable importance in projection (VIP) value calculated with OPLS-DA. One-way ANOVA was performed using SPSS 16.0 (Chicago, IL, USA). 


\section{Results and Discussion}

3.1. EO Yields of DHC and FHC and Their Two Harvest Seasons. The EO yields (\%, v/w) of DHC and FHC and their two harvest seasons (DHC1 and DHC2; FHC1 and FHC2) are shown in Figure 1. The results showed that the EO yield of DHC $(0.038 \pm 0.029 \%)$ was significantly lower than that of FHC $(0.076 \pm 0.030 \%)(p<0.05)$. This was probably due to the loss or decomposition of various volatile components during the drying process [1]. On the other hand, between the two harvest seasons, the EO yields of DHC1 $(0.044 \pm 0.029 \%)$ were a little higher than that of DHC2 $(0.036 \pm 0.028 \%)$, and so were FHC1 $(0.084 \pm 0.026 \%)$ and FHC2 $(0.067 \pm 0.033 \%)$, indicating that the content of EO was higher in summer than that in autumn. One potential explanation for this finding is that the flowering season of $\mathrm{HC}$ was in summer and flowers could produce the most oil [9].

3.2. Characterization and Classification of Chemical Profiles by GC-MS. The EOs extracted from 44 samples of DHC and FHC at two harvest seasons were comprehensively analyzed by GC-MS. According to total ion chromatograms (TIC), the chemical components of DHC and FHC were similar in general but still had some differences. Visually, the quantity and content of peaks of DHC from $42 \mathrm{~min}$ to 47 min were higher than of FHC (Figure 2). The chemical constituents of EOs of samples were characterized according to the MS-DIAL and NIST17.0 Mass Spectra Database. Altogether, 69 common constituents were identified, as listed in Table 1 . The oils were predominantly composed of two categories of chemical constituents, i.e., nonterpene compounds (aliphatic compounds and aromatic compounds) and terpenoids (terpene hydrocarbons including monoterpene hydrocarbons, sesquiterpene hydrocarbons, diterpene hydrocarbons, and oxygenated terpenoids). The average percentage of the terpenoids and nonterpene compounds were almost the same in both DHC and FHC, in which the content of aliphatic compounds and monoterpene hydrocarbons were present in relatively high amounts (more than $50 \%$ and $30 \%$, respectively). The results were consistent with the previous reports $[3,10]$. Moreover, the contents of the three most abundant components (2-undecanone, $\beta$-myrcene, and $\beta$-pinene) account for about $70 \%$ in both DHC and FHC and in the two harvest seasons of DHC and FHC. Besides, 2-undecanone was the most abundant component, which was the quality control component of HC in Chinese Pharmacopoeia.

However, it was worth noting that oxygenated terpenoids in DHC (9.72\%) were higher than in FHC (6.55\%), whereas terpenoids in DHC (31.95\%) were lower than in FHC (35.79\%). One potential explanation is the loss of the terpenoids or its oxidation to oxygenated terpenoids during the drying process $[11,12]$. Moreover, the content of some chemical constituents had intuitive differences and varied with DHC and FHC and two harvest seasons. The contents of the components such as $\alpha$-pinene, (1S)-(-)- $\beta$-pinene, $\beta$-myrcene,(E)-3,7-dimethyl-2,6-octadien-1-ol,

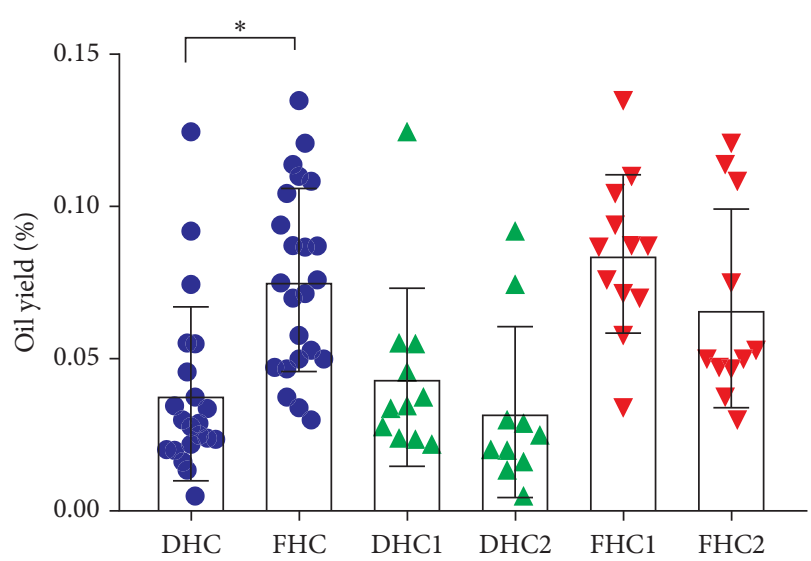

Figure 1: The EO yields (\%, v/w) of twenty-one batches of DHC and twenty-three batches of FHC and their two harvest seasons (DHC1 and DHC2; FHC1 and FHC2). Data were presented as mean \pm SD. ${ }^{*} p<0.05$, DHC compared with FHC.

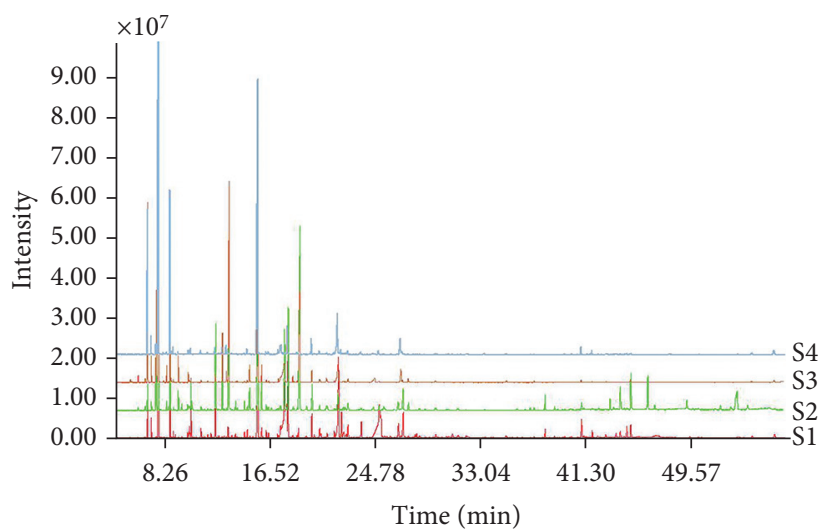

Figure 2: Representative total ion chromatograms of EOs of DHC1, DHC2, FHC1, and FHC2. S1: DHC1; S2: DHC2; S3: FHC1; S4: FHC2.

$\beta$-phellandrene $(2.18 \pm 1.15 \%, \quad 1.64 \pm 1.79 \%, \quad 18.36 \pm 7.64$ , $2.44 \pm 1.99 \%$, and $1.82 \pm 2.23 \%$, respectively) were higher in FHC oil than in DHC oil $(0.63 \pm 0.44 \%, 0.77 \pm 1.05 \%$, $17.68 \pm 12.99 \%, 1.55 \pm 1.73 \%$, and $0.53 \pm 1.51 \%$, respectively), while the contents of 1-nonanol, geranyl acetate,2-tridecanone, tetradecanal, and bornyl acetate were higher in DHC oil $(3.10 \pm 1.50 \%, 2.65 \pm 1.80 \%, 2.29 \pm 2.16 \%, 1.48 \pm 1.19 \%$, and $1.89 \pm 0.92 \%$, respectively) than in FHC oil $(0.49 \pm 0.55 \%, 0.75 \pm 0.46 \%, 1.18 \pm 1.10 \%, 0.82 \pm 0.38 \%$, and $1.28 \pm 0.26 \%$, respectively). Compared with the results of DHC and FHC, the differences of oils between two harvest seasons (DHC1 and DHC2; FHC1 and FHC2) were not so obvious. Some constituents such as (E)-3,7-dimethyl-2,6octadien-1-ol and $\beta$-phellandrene in FHC1 oil (3.04 $\pm 2.44 \%$ and $2.82 \pm 2.65 \%$, respectively) were higher than in FHC2 oil $(1.80 \pm 1.15 \%$ and $0.73 \pm 0.83 \%$, respectively), while decanal and dodecanal were lower in FHC1 $(1.30 \pm 3.26 \%$ and $0.04 \pm 0.03 \%$, respectively) than in FHC2 $(4.04 \pm 7.08 \%$ and $0.58 \pm 1.58 \%$, respectively). The percentage composition of $\beta$-phellandrene in DHC1 $(0.99 \pm 2.02 \%)$ was higher than in DHC2 $(0.03 \pm 0.05 \%)$, whereas phytol was lower in DHC1 
TABLE 1: Average relative content of sixty-nine common volatile constituents of DHC and FHC at two harvest seasons.

\begin{tabular}{|c|c|c|c|c|c|c|c|c|c|}
\hline \multirow[b]{2}{*}{ No. } & \multirow[b]{2}{*}{ Name/class } & \multirow{2}{*}{$\begin{array}{l}\mathrm{RT} \\
(\mathrm{min})\end{array}$} & \multirow{2}{*}{$\begin{array}{l}\text { Similarity } \\
(\%)\end{array}$} & \multicolumn{6}{|c|}{ Average relative content (\%) } \\
\hline & & & & $\begin{array}{c}\text { DHC } \\
(n=21)\end{array}$ & $\mathrm{FHC}(n=23)$ & $\begin{array}{c}\text { DHC1 } \\
(n=11)\end{array}$ & $\begin{array}{c}\text { DHC2 } \\
(n=10)\end{array}$ & $\begin{array}{l}\text { FHC1 } \\
(n=12)\end{array}$ & $\begin{array}{c}\text { FHC2 } \\
(n=11)\end{array}$ \\
\hline \multicolumn{10}{|c|}{ Aliphatic compounds } \\
\hline 1 & Nonane & 6.47 & 99 & $0.02(0.02)$ & $0.02(0.03)$ & $0.02(0.01)$ & $0.03(0.03)$ & $0.02(0.03)$ & $0.02(0.02)$ \\
\hline 2 & 2-Nonanone & 10.38 & 96 & $0.06(0.10)$ & $0.02(0.02)$ & $0.09(0.13)$ & $0.02(0.01)$ & $0.01(0.01)$ & $0.03(0.03)$ \\
\hline 3 & Undecane & 10.59 & 98 & $0.04(0.03)$ & $0.02(0.01)$ & $0.04(0.04)$ & $0.04(0.03)$ & $0.01(0.01)$ & $0.02(0.01)$ \\
\hline 4 & Nonanal & 10.75 & 90 & $0.67(0.68)$ & $0.07(0.04)$ & $0.81(0.85)$ & $0.50(0.39)$ & $0.05(0.03)$ & $0.08(0.05)$ \\
\hline 5 & 1-Nonanol & 12.75 & 99 & $3.10(1.50)$ & $0.49(0.55)$ & $2.73(1.15)$ & $3.51(1.80)$ & $0.31(0.21)$ & $0.69(0.73)$ \\
\hline 6 & Decanal & 13.88 & 99 & $2.68(6.35)$ & $2.61(5.48)$ & $2.55(7.32)$ & $2.82(5.47)$ & $1.30(3.26)$ & $4.04(7.08)$ \\
\hline 7 & 1-Decanol & 15.53 & 99 & $0.22(0.55)$ & $0.14(0.20)$ & $0.29(0.75)$ & $0.13(0.13)$ & $0.07(0.08)$ & $0.22(0.27)$ \\
\hline 8 & 2-Undecanone & 16.25 & 92 & $44.92(27.15)$ & $48.49(15.48)$ & $44.23(30.29)$ & $45.67(24.73)$ & $45.94(13.18)$ & $51.27(17.87)$ \\
\hline 9 & Undecanal & 16.5 & 99 & $0.19(0.40)$ & $0.09(0.22)$ & $0.25(0.54)$ & $0.14(0.16)$ & $0.02(0.04)$ & $0.17(0.31)$ \\
\hline 10 & 1-Undecanol & 18.31 & 99 & $0.60(0.83)$ & $0.17(0.22)$ & $0.68(0.97)$ & $0.52(0.70)$ & $0.17(0.12)$ & $0.18(0.30)$ \\
\hline 11 & 2-Dodecanone & 19.01 & 91 & $0.30(0.31)$ & $0.22(0.17)$ & $0.28(0.28)$ & $0.31(0.36)$ & $0.17(0.12)$ & $0.28(0.21)$ \\
\hline 12 & $n$-Decanoic acid & 19.02 & 98 & $0.08(0.05)$ & $0.04(0.03)$ & $0.06(0.03)$ & $0.10(0.07)$ & $0.02(0.01)$ & $0.05(0.03)$ \\
\hline 13 & Dodecanal & 19.48 & 99 & $0.12(0.17)$ & $0.30(1.10)$ & $0.07(0.06)$ & $0.17(0.23)$ & $0.04(0.03)$ & $0.58(1.58)$ \\
\hline 14 & Dodecanol & 21.76 & 99 & $0.10(0.19)$ & $0.04(0.07)$ & $0.12(0.26)$ & $0.07(0.04)$ & $0.02(0.02)$ & $0.06(0.09)$ \\
\hline 15 & Vinyl decanoate & 21.8 & 89 & $0.10(0.05)$ & $0.05(0.03)$ & $0.08(0.04)$ & $0.11(0.05)$ & $0.04(0.01)$ & $0.06(0.03)$ \\
\hline 16 & 4-Tridecanone & 22.66 & 89 & $0.55(0.56)$ & $1.78(1.11)$ & $0.76(0.55)$ & $0.32(0.49)$ & $2.03(0.79)$ & $1.52(1.37)$ \\
\hline 17 & 2-Tridecanone & 22.67 & 92 & $2.29(2.16)$ & $1.18(1.10)$ & $2.58(2$ & 1.9 & $1.41(1.41)$ & $0.92(0$ \\
\hline 18 & Ethyl decanoate & 27.81 & 82 & $0.03(0.02)$ & $0.80(0.46)$ & $0.04(0.02)$ & $0.02(0.02)$ & $0.81(0.26)$ & $0.79(0.63)$ \\
\hline 19 & Tetradecanal & 28.4 & 99 & $1.48(1.19)$ & $0.82(0.38)$ & $1.27(1.12)$ & $1.72(1.27)$ & $0.78(0.39)$ & $0.87(0.37)$ \\
\hline 20 & Isopentyl decanoate & 36.36 & 78 & $0.06(0.04)$ & $0.11(0.07)$ & $0.06(0.02)$ & $0.06(0.06)$ & $0.09(0.05)$ & $0.13(0.09)$ \\
\hline 21 & $n$-Hexadecanoic acid & 42.62 & 9 & 0.09 & 0.01 & 0.07 & 0 . & $0.01(0$ & $2(0.01)$ \\
\hline 22 & Octadecanol & 45.57 & 99 & $0.36(0.39)$ & $0.04(0.03)$ & & $0.58(0.48)$ & $0.02(0.01)$ & $0.04)$ \\
\hline \multicolumn{10}{|c|}{ Aromatic compounds } \\
\hline 23 & Benzeneacetaldehyde & 9.35 & 92 & $0.06(0.05)$ & $0.04(0.04)$ & $0.05(0.03)$ & $0.08(0.06)$ & $0.04(0.05)$ & $0.03(0.02)$ \\
\hline 24 & $\begin{array}{l}\text { 4-Isopropyl-1- } \\
\text { methylcyclohex-2-enol }\end{array}$ & 11.55 & 96 & $0.08(0.10)$ & $0.06(0.08)$ & $0.07(0.06)$ & $0.10(0.13)$ & $0.08(0.10)$ & $0.05(0.05)$ \\
\hline 25 & $\begin{array}{l}\text { 2-Butanone, } 4-(2,6,6- \\
\text { trimethyl-1,3- } \\
\text { cyclohexadien-1-yl)- }\end{array}$ & 19.96 & 85 & $0.05(0.05)$ & $0.02(0.02)$ & $0.03(0.02)$ & $0.08(0.06)$ & $0.01(0.01)$ & $0.03(0.02)$ \\
\hline 26 & $\begin{array}{l}\text { 1,4-Di-Tert- } \\
\text { Butylbenzene }\end{array}$ & 21.75 & 80 & $0.10(0.12)$ & $0.03(0.04)$ & $0.11(0.17)$ & $0.08(0.05)$ & $0.02(0.01)$ & $0.05(0.06)$ \\
\hline \multicolumn{10}{|c|}{ Monoterpene hydrocarbons } \\
\hline 27 & $\begin{array}{l}\text { Bicyclo[3.1.0]hex-2- } \\
\text { ene,2-methyl-5-(1- } \\
\text { methylethyl)- }\end{array}$ & 7.04 & 92 & $0.04(0.05)$ & $0.06(0.06)$ & $0.03(0.04)$ & $0.05(0.06)$ & $0.05(0.06)$ & $0.07(0.07)$ \\
\hline 28 & $\alpha$-Pinene & 7.2 & 9 & $0.63(0.44)$ & $2.18(1.15)$ & 0.6 & & & $2.11(1.22)$ \\
\hline 29 & Camphene & 7.54 & 99 & & & & & & \\
\hline 30 & $(1 S)-(-)-\beta$-Pinene & 7.92 & 91 & $0.77(1.05)$ & $1.64(1.79)$ & $0.62(0.95)$ & $0.95(1.17)$ & $1.62(1.97)$ & $1.67(1.65)$ \\
\hline 31 & $\beta$-Pinene & 8.09 & 99 & $9.95(7.16)$ & $8.67(3.43)$ & $10.59(7.40)$ & $9.25(7.21)$ & $9.23(3.93)$ & $8.07(2.85)$ \\
\hline 32 & $\beta$-Myrcene & 8.14 & 99 & $17.68(12.99)$ & $18.36(7.64)$ & $18.85(13.44)$ & $16.39(13.06)$ & $21.33(8.72)$ & $15.12(5.58)$ \\
\hline 33 & $\alpha$-Phellandrene & 8.54 & 98 & $0.02(0.02)$ & $0.03(0.02)$ & $0.02(0.02)$ & $0.02(0.01)$ & $0.03(0.01)$ & $0.04(0.03)$ \\
\hline 34 & $\alpha$-Terpinene & 8.78 & 99 & $0.06(0.08)$ & $0.16(0.17)$ & $0.05(0.07)$ & $0.08(0.08)$ & $0.13(0.14)$ & $0.19(0.20)$ \\
\hline 35 & $\begin{array}{l}\text { 1-Isopropyl-4- } \\
\text { Methylbenzene }\end{array}$ & 8.94 & 95 & $0.09(0.10)$ & $0.08(0.13)$ & $0.09(0.11)$ & $0.09(0.10)$ & $0.06(0.13)$ & $0.10(0.14)$ \\
\hline 36 & Limonene & 9.06 & 98 & $0.37(0.22)$ & 1.3 & 0.36 & 0.38 & 1.3 & 1.3 \\
\hline 37 & $\beta$-Phellandrene & 9.13 & 93 & $0.53(1.51)$ & $1.82(2.23)$ & $0.99(2.02)$ & $0.03(0.05)$ & $2.82(2.65)$ & $0.73(0.83)$ \\
\hline 38 & $\begin{array}{c}\text { 1,3,6-Octatriene, 3,7- } \\
\text { dimethyl-, }(\mathrm{Z})-\end{array}$ & 9.31 & 96 & $0.07(0.06)$ & $0.10(0.09)$ & $0.06(0.05)$ & $0.08(0.07)$ & $0.11(0.10)$ & $0.09(0.07)$ \\
\hline 39 & $\begin{array}{c}\text { 2-Octene, 2-methyl-6- } \\
\text { methylene- }\end{array}$ & 9.47 & 80 & $.08(0.07)$ & $0.01(0.01)$ & $0.09(0.09)$ & $0.07(0.05)$ & $0.01(0.01)$ & $0.02(0.01)$ \\
\hline 40 & Gamma-terpinene & 9.73 & 99 & $0.22(0.28)$ & $0.44(0.47)$ & $0.19(0.27)$ & $0.25(0.29)$ & $0.32(0.36)$ & $0.56(0.56)$ \\
\hline 41 & Terpinolene & 10.54 & 96 & $0.05(0.04)$ & $0.11(0.08)$ & $0.05(0.04)$ & $0.05(0.04)$ & $0.09(0.07)$ & $0.14(0.10)$ \\
\hline \multicolumn{10}{|c|}{ Sesquiterpene hydrocarbons } \\
\hline 42 & Caryophyllene & 20.56 & 99 & $(0.13)$ & $(0.08)$ & 10) & 0.3 & $0.13(0.04)$ & $0.19(0.11)$ \\
\hline 43 & $\alpha$-Guaiene & 22.37 & 91 & $0.07(0.05)$ & $0.03(0.01)$ & $0.07(0.05)$ & $0.08(0.05)$ & $0.03(0.01)$ & $0.03(0.02)$ \\
\hline
\end{tabular}


TABle 1: Continued.

\begin{tabular}{|c|c|c|c|c|c|c|c|c|c|}
\hline \multirow[b]{2}{*}{ No. } & \multirow[b]{2}{*}{ Name/class } & \multirow[b]{2}{*}{$\begin{array}{c}\mathrm{RT} \\
(\mathrm{min})\end{array}$} & \multirow[b]{2}{*}{$\begin{array}{l}\text { Similarity } \\
(\%)\end{array}$} & \multicolumn{6}{|c|}{ Average relative content (\%) } \\
\hline & & & & $\begin{array}{c}\text { DHC } \\
(n=21)\end{array}$ & $\mathrm{FHC}(n=23)$ & $\begin{array}{l}\text { DHC1 } \\
(n=11)\end{array}$ & $\begin{array}{c}\mathrm{DHC} 2 \\
(n=10)\end{array}$ & $\begin{array}{c}\text { FHC1 } \\
(n=12)\end{array}$ & $\begin{array}{l}\text { FHC2 } \\
(n=11)\end{array}$ \\
\hline 44 & 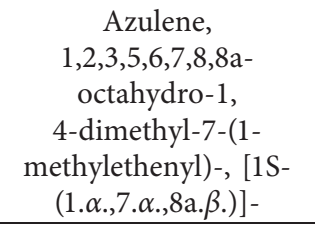 & 23.5 & 91 & $0.14(0.08)$ & $0.07(0.04)$ & $0.13(0.07)$ & $0.16(0.080$ & $0.07(0.05)$ & $0.07(0.03)$ \\
\hline \multicolumn{10}{|c|}{ Diterpene hydrocarbons } \\
\hline 45 & $\begin{array}{c}\text { 3-Methylene-7,11,15- } \\
\text { Trimethyl-1,6,10,14- } \\
\text { Hexadecatetraene }\end{array}$ & 21.19 & 90 & $0.19(0.12)$ & $0.07(0.04)$ & $0.17(0.0)$ & $0.21(0.13)$ & $0.07(0.04)$ & $0.07(0.03)$ \\
\hline 46 & m-Camphorene & 42.42 & 95 & $0.19(0.18)$ & $0.11(0.08)$ & $0.19(0.15)$ & $0.19(0.21)$ & $0.12(0.09)$ & $0.09(0.06)$ \\
\hline 47 & p-Camphorene & 43.3 & 94 & $0.09(0.09)$ & $0.05(0.04)$ & $0.08(0.07)$ & $0.10(0.11)$ & $0.06(0.05)$ & $0.04(0.04)$ \\
\hline 48 & (E)- $\beta$-Farnesene & 46.1 & 78 & $0.26(0.27)$ & $0.03(0.02)$ & $0.22(0.20)$ & $0.29(0.33)$ & $0.02(0.01)$ & $0.04(0.02)$ \\
\hline \multicolumn{10}{|c|}{ Oxygenated monoterpenes } \\
\hline 49 & $\begin{array}{l}\text { (E)-3,7-Dimethyl-2,6- } \\
\text { octadien-1-ol } \\
\text { (3E,5 E)-2,6- }\end{array}$ & 9.06 & 90 & $1.55(1.73)$ & $2.44(1.99)$ & $1.59(1.78)$ & $1.51(1.78)$ & $3.04(2.44)$ & $1.80(1.15)$ \\
\hline 50 & $\begin{array}{l}\text { Dimethylocta-3,5,7- } \\
\text { trien-2-ol }\end{array}$ & 10.62 & 85 & $0.07(0.04)$ & $0.03(0.02)$ & $0.06(0.04)$ & $0.09(0.05)$ & $0.03(0.02)$ & $0.04(0.03)$ \\
\hline 51 & $\begin{array}{l}\text { 2-Methyl-6- } \\
\text { Methylene-2,7- } \\
\text { octadien-4-ol }\end{array}$ & 12.06 & 92 & $0.07(0.05)$ & $0.04(0.03)$ & $0.06(0.04)$ & $0.08(0.06)$ & $0.03(0.02)$ & $0.05(0.03)$ \\
\hline 52 & Isoborneol & 13.03 & 96 & $0.09(0.07)$ & $0.05(0.02)$ & $0.10(0.07)$ & $0.07(0.06)$ & $0.04(0.01)$ & $0.06(0.02)$ \\
\hline 53 & 4-Terpinenol & 13.31 & 99 & $0.52(0.55)$ & $0.93(1.06)$ & $0.53(0.61)$ & $0.51(0$ & $0.65(0.77)$ & $1.25(1.26)$ \\
\hline 54 & Neral & 13.45 & 8 & $0.02(0.02)$ & $0.02(0.03)$ & $0.02(0.01)$ & $0.02(0.030$ & $0.02(0.03)$ & $0.01(0.01)$ \\
\hline 55 & $\alpha$-Terpineol & 13.63 & 99 & $0.06(0.03)$ & $0.21(0.13)$ & $0.07(0.03)$ & $0.06(0.02)$ & $0.17(0.09)$ & $0.26(0.16)$ \\
\hline 56 & $\begin{array}{c}\text { 2-Cyclohexen-1-ol, 3- } \\
\text { methyl-6-(1- } \\
\text { methylethyl)-, trans- }\end{array}$ & 14.06 & 91 & $0.06(0.04)$ & $0.03(0.02)$ & $0.06(0.05)$ & $0.05(0.04)$ & $0.03(0.02)$ & $0.04(0.03)$ \\
\hline 57 & $\beta$-Cyclocitral & 14.36 & 91 & $0.12(0.06)$ & $0.01(0.01)$ & $0.09(0.05)$ & $0.15(0.06)$ & $0.01(0.00)$ & $0.02(0.01)$ \\
\hline 58 & $\begin{array}{l}\text { 3,7-Dimethyl-2,6- } \\
\text { octadien-1-ol(trans) }\end{array}$ & 15.1 & 99 & $0.18(0.11)$ & $0.03(0.01)$ & $0.18(0.09)$ & $0.17(0.13)$ & $0.02(0.01)$ & $0.03(0.02)$ \\
\hline 59 & $\begin{array}{l}\text { 4-(1-Methylethenyl) } \\
\text { cyclohexene-1- } \\
\text { methanol }\end{array}$ & 15.33 & 86 & $0.08(0.06)$ & $0.04(0.03)$ & $0.05(0.03)$ & $0.12(0.07)$ & $0.03(0.02)$ & $0.05(0.03)$ \\
\hline 60 & Bornyl acetate & 16.18 & 92 & $1.89(0.92)$ & $1.28(0.26)$ & $1.85(1.06)$ & $1.93(0.80)$ & $1.27(0.22)$ & $1.30(0.31)$ \\
\hline 61 & $\begin{array}{l}\alpha \text {-Terpinyl acetate } \\
\text { 4-Hexen-1-ol, 5- }\end{array}$ & 17.86 & 85 & $0.06(0.03)$ & $0.07(0.04)$ & $0.05(0.03)$ & $0.06(0.03)$ & $0.06(0.04)$ & $0.08(0.04)$ \\
\hline 62 & $\begin{array}{l}\text { methyl-2-(1- } \\
\text { methylethenyl)-, } \\
\text { acetate }\end{array}$ & 18.02 & 90 & $0.11(0.08)$ & $0.06(0.03)$ & $0.09(0.07)$ & $0.12(0.08)$ & $0.04(0.01)$ & $0.07(0.04)$ \\
\hline 63 & Geranyl acetate & 18.62 & 98 & $2.65(1.80)$ & $0.75(0.46)$ & $2.23(0.94)$ & $3.12(2.41)$ & $0.59(0.30)$ & $0.91(0.56)$ \\
\hline 64 & $\begin{array}{l}\text { 2,6-Octadien-1-ol, 3,7- } \\
\text { dimethyl-, acetate, (Z)- }\end{array}$ & 18.63 & 94 & $0.28(0.21)$ & $0.15(0.10)$ & $0.22(0.12)$ & $0.34(0.27)$ & $0.08(0.03)$ & $0.23(0.09)$ \\
\hline \multicolumn{10}{|c|}{ Oxygenated sesquiterpenes } \\
\hline 65 & $\begin{array}{l}\text { trans-Nerolidyl } \\
\text { formate }\end{array}$ & 26.01 & 97 & $0.34(0.35)$ & $0.07(0.06)$ & $0.32(0.27)$ & $0.36(0.450$ & $0.07(0.05)$ & $0.07(0.06)$ \\
\hline 66 & $\begin{array}{l}\text { Caryophyllene oxide } \\
\text { Oxygenated diterpenes }\end{array}$ & 27.95 & 96 & $0.37(0.25)$ & $0.04(0.02)$ & $0.34(0.25)$ & $0.39(0.27)$ & $0.03(0.01)$ & $0.05(0.03)$ \\
\hline 67 & $\begin{array}{c}\text { 5,9,13,17-Tetramethyl } \\
4,8,12,16- \\
\text { Octadecatetraenoic } \\
\text { acid }\end{array}$ & 34.51 & 88 & $0.08(0.11)$ & $0.03(0.04)$ & $0.06(0.05)$ & $0.11(0.15)$ & $0.02(0.01)$ & $0.04(0.06)$ \\
\hline 68 & Phytol & 46.42 & 96 & $1.06(0.94)$ & $0.19(0.21)$ & $0.54(0.20)$ & $1.63(1.11)$ & $0.07(0.03)$ & $0.33(0.24)$ \\
\hline
\end{tabular}


TABLE 1: Continued.

\begin{tabular}{|c|c|c|c|c|c|c|c|c|c|}
\hline \multirow[b]{2}{*}{ No. } & \multirow[b]{2}{*}{ Name/class } & \multirow{2}{*}{$\begin{array}{c}\mathrm{RT} \\
(\mathrm{min})\end{array}$} & \multirow{2}{*}{$\begin{array}{c}\text { Similarity } \\
(\%)\end{array}$} & \multicolumn{6}{|c|}{ Average relative content (\%) } \\
\hline & & & & $\begin{array}{c}\text { DHC } \\
(n=21)\end{array}$ & FHC $(n=23)$ & $\begin{array}{c}\text { DHC1 } \\
(n=11)\end{array}$ & $\begin{array}{c}\text { DHC2 } \\
(n=10)\end{array}$ & $\begin{array}{c}\text { FHC1 } \\
(n=12)\end{array}$ & $\begin{array}{c}\text { FHC2 } \\
(n=11)\end{array}$ \\
\hline 69 & $\begin{array}{c}1,6,10,14- \\
\text { Hexadecatetraen-3- } \\
\text { ol,3,7,11,15- } \\
\text { tetramethyl-, (E,E)- } \\
\text { Total identified (\%) }\end{array}$ & 56.29 & 81 & $0.07(0.05)$ & $0.06(0.03)$ & $0.05(0.03)$ & $0.08(0.07)$ & $0.04(0.01)$ & $0.08(0.03)$ \\
\hline \multicolumn{9}{|c|}{ Class composition } & $97.67(2.70)$ \\
\hline & $\begin{array}{l}\text { Nonterpene } \\
\text { compounds }\end{array}$ & & & $58.34(23.28)$ & $57.66(12.39)$ & $57.51(25.68)$ & $59.25(21.67)$ & $53.51(12.04)$ & $62.19(11.62)$ \\
\hline & $\begin{array}{l}\text { Aliphatic } \\
\text { compounds }\end{array}$ & & & $58.04(23.34)$ & $57.50(12.47)$ & $57.25(25.74)$ & $58.91(21.75)$ & $53.36(12.15)$ & $62.03(11.67)$ \\
\hline & $\begin{array}{l}\text { Aromatic } \\
\text { compounds }\end{array}$ & & & $0.30(0.18)$ & $0.15(0.11)$ & $0.25(0.18)$ & $0.34(0.17)$ & $0.15(0.13)$ & $0.16(0.09)$ \\
\hline & Terpenoids & & & $41.66(23.28)$ & $42.34(12.39)$ & $42.49(25.68)$ & $40.75(21.67)$ & $46.49(12.04)$ & $37.81(11.62)$ \\
\hline & $\begin{array}{c}\text { Terpene } \\
\text { hydrocarbons }\end{array}$ & & & $31.95(21.79)$ & $35.79(10.95)$ & $33.93(23.15)$ & $29.77(21.20)$ & $40.13(10.06)$ & $31.05(10.25)$ \\
\hline & $\begin{array}{l}\text { Monoterpene } \\
\text { hydrocarbons }\end{array}$ & & & $30.74(21.40)$ & $35.27(10.85)$ & $32.84(22.53)$ & $28.42(21.04)$ & $39.62(9.88)$ & $30.52(10.21)$ \\
\hline & $\begin{array}{l}\text { Sesquiterpene } \\
\text { hydrocarbons }\end{array}$ & & & $0.48(0.22)$ & $0.26(0.11)$ & $0.42(0.19)$ & $0.55(0.24)$ & $0.23(0.08)$ & $0.29(0.13)$ \\
\hline & $\begin{array}{c}\text { Diterpene } \\
\text { hydrocarbons }\end{array}$ & & & $0.73(0.62)$ & $0.26(0.17)$ & $0.66(0.51)$ & $0.80(0.75)$ & $0.28(0.19)$ & $0.24(0.14)$ \\
\hline & $\begin{array}{l}\text { Oxygenated } \\
\text { terpenes }\end{array}$ & & & $9.72(4.07)$ & $6.55(2.09)$ & $8.56(3.21)$ & $10.99(4.69)$ & $6.36(2.33)$ & $6.76(1.88)$ \\
\hline & Oil yield $(\%, v / w)$ & & & $0.04(0.03)$ & $0.08(0.03)$ & $0.04(0.03)$ & $0.03(0.03)$ & $0.08(0.03)$ & $0.07(0.03)$ \\
\hline & Total identified (\%) & & & $95.62(4.63)$ & $98.41(2.01)$ & $94.88(5.12)$ & $96.43(4.13)$ & $99.09(0.61)$ & $97.67(2.70)$ \\
\hline
\end{tabular}

Note: RT represents retention time. Data were presented as mean (SD).

$(0.54 \pm 0.20 \%)$ than in DHC2 $(1.63 \pm 1.11 \%)$. These differences between the samples might be attributed to the medicinal parts, drying process, and collection seasons $[1,3]$. In order to further explore the distinctions between DHC and FHC and their two harvest seasons, chemometric methods were utilized.

3.3. Heatmap and HCA and PCA Analysis. To make the overall discrepancies in the chemical profiles of DHC and FHC oils more externally, heatmap and HCA analysis were further performed to explore the prominent distinctions between DHC and FHC. Heatmap is one of the most popular bioinformatic graphic displays for data visualization by color intensity. HCA is a clustering technique that measures either the difference or the similarity among the samples to be clustered. Based on the relative contents of each constituent in the EOs of the samples, the position of various samples will be crudely redistributed, and samples with close similarities will be classified into the same group by HCA. As illustrated in Figure 3, the blue box indicated that the content was lower, while the red box showed a greater content than the average level of the sample. It was noted that forty-four batches of samples were apparently classified into two categories; i.e., the first group included twenty-one batches of DHC and the second group twenty-three batches of FHC. Nevertheless, eleven batches of DHC1 and ten batches of DHC2 could not be well clustered; neither were twelve batches of FHC1 nor eleven batches of FHC2. In other words, although there was significant difference in chemical composition between DHC and FHC, the difference between DHC1 and DHC2 and FHC1 and FHC2 was not obvious.

Moreover, another unsupervised PCA approach was employed to provide more information about the discrimination of DHC and FHC (Figure 4(a)). The results showed that $R^{2} X=0.773$ and $Q^{2}=0.519$, which indicated that the model was reliable and had good predictive ability. The score plot showed that the DHC (both blue and green spots) and FHC (both red and yellow spots) were clearly separated. However, DHC1 (green spots) and DHC2 (blue spots) and FHC1 (red spots) and FHC2 (yellow spots) could not be classified obviously. The result was consistent with heatmap and HCA analysis. In general, HCA and PCA are both good classification tools that distinguished DHC and FHC. Still, they could not well classify the samples at two harvest seasons and could not find out the major chemical differential components between them. To find their chemical markers for discrimination, OPLS-DA analysis was applied.

3.4. OPLS-DA Analysis and Identification of Chemical Markers. To further distinguish the major variations responsible for the differentiation and find the chemical markers, a supervised OPLS-DA approach was constructed to maximize sample separation. As indicated in Figure 4(b), the samples of DHC and FHC were distributed on two sides 


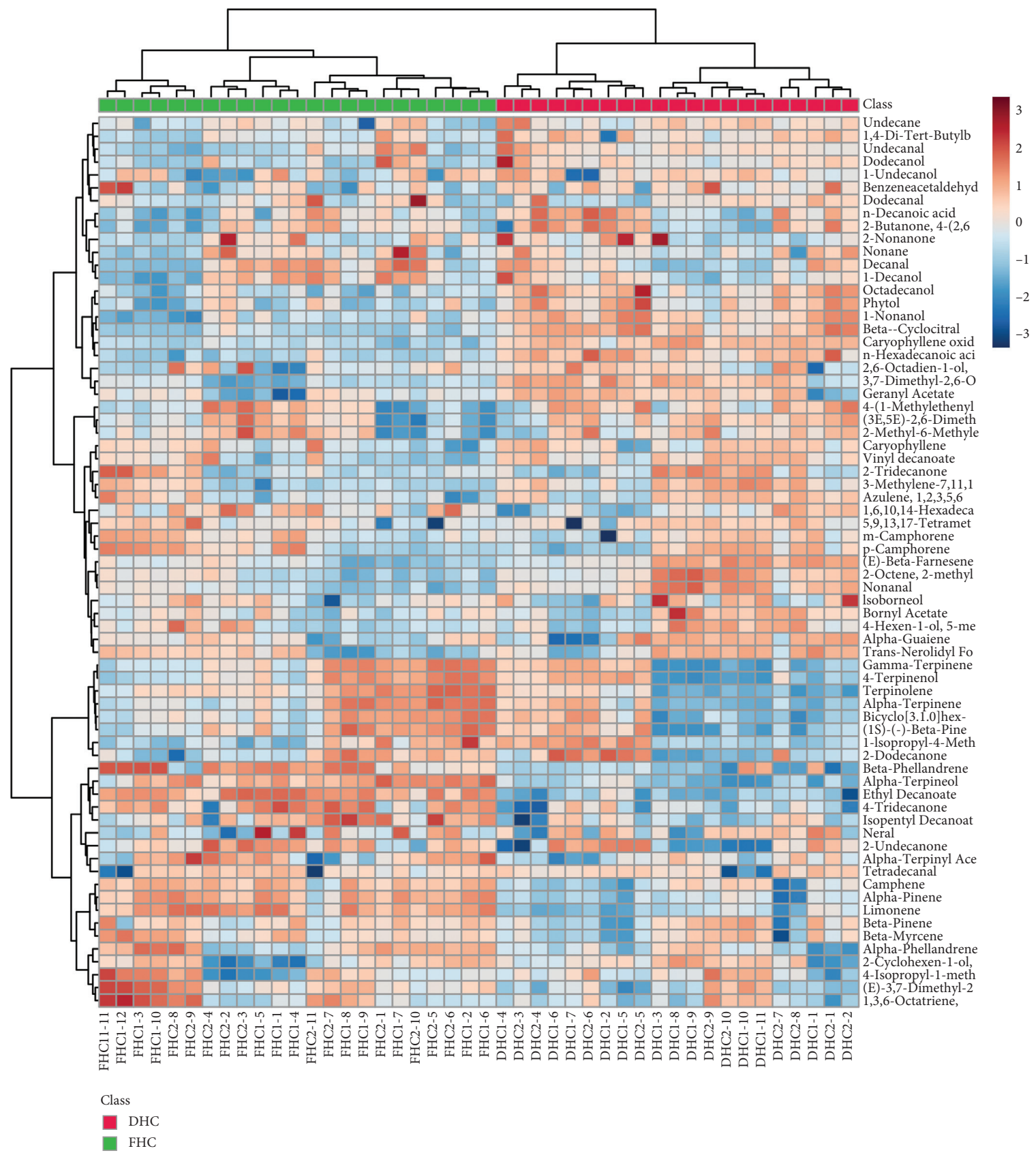

Figure 3: Heatmap analysis and HCA analysis of twenty-one batches of DHC and twenty-three batches of FHC at two harvest seasons. The different degrees of color clearly indicate the relationship between these chemical components in different samples.

evidently. The variation in $\mathrm{X}$ (R2Xcum) was 0.658 and the variation in $\mathrm{Y}$ (R2Ycum) was 0.975 , predicting $91.9 \%$ of the variation in response to $\mathrm{Y}(\mathrm{Q} 2 \mathrm{cum}=0.919)$. The value of these parameters demonstrated that the model had high reliability and predictive abilities. To validate the model, 200 times permutation tests were performed (Figure 4(c)). Moreover, the $p$ value and VIP value of the 69 common constituents were obtained. The constituents with VIP value $>1.5$ and $p$ value $<0.01$ were regarded as potential chemical markers responsible for the distinctions. As a result, the eleven components including $\alpha$-pinene, limonene, $\beta$-phellandrene, $\alpha$-terpineol, 4-tridecanone, ethyl decanoate, nonanal, 1-nonanol, $\beta$-cyclocitral, $\mathrm{n}$-hexadecanoic acid, and octadecanol had significant qualitative differences, which were screened as chemical markers between DHC and FHC.

Regarding the two harvest seasons of DHC, the samples of DHC1 and DHC2 were clearly classified and aggregated on the scatter plot (Figure $4(\mathrm{~d})$ ). The variation in $\mathrm{X}$ (R2Xcum) was 0.617 and the variation in Y (R2Ycum) was 0.974 , predicting $70.4 \%$ of the variation in response to $\mathrm{Y}$ $(\mathrm{Q} 2$ cum $=0.704)$. Using the same screening criteria as used above, two components, including 4-tridecanone and ethyl decanoate, were identified as chemical markers for DHC1 and DHC2. In addition, the samples of FHC1 and FHC2 


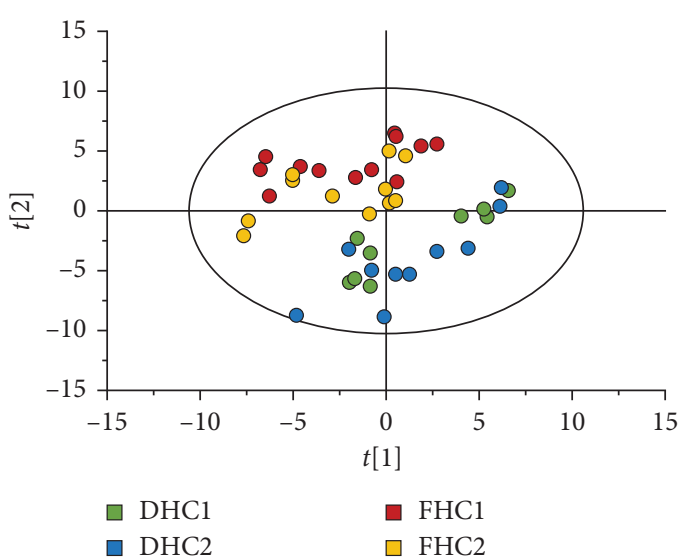

(a)

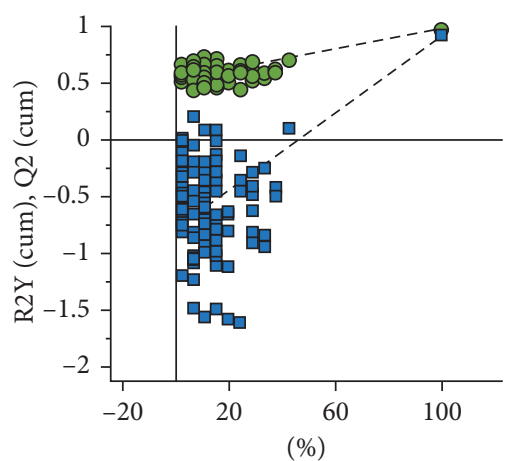

- R2Y (cum)

- Q2 (cum)

(c)

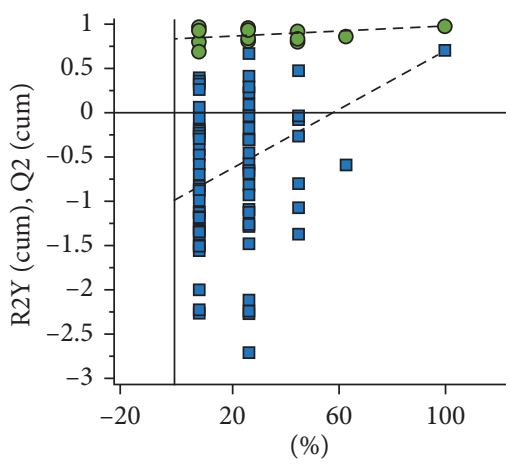

- R2Y (cum)

घ $\mathrm{Q} 2$ (cum)

(e)

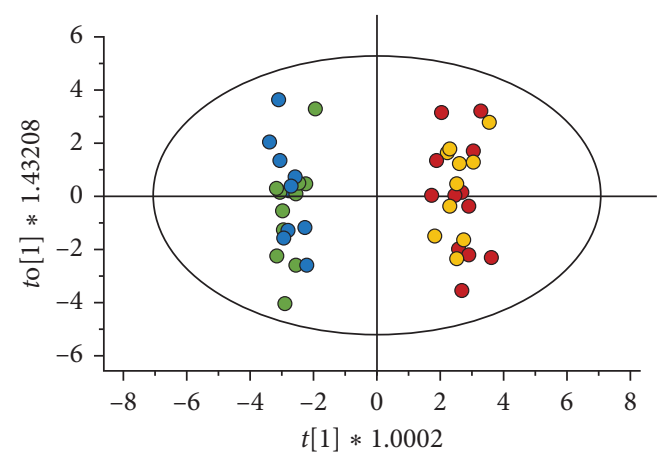

$\square$ DHC1

- DHC2

- $\mathrm{FHC1}$

$\square$ FHC2

(b)

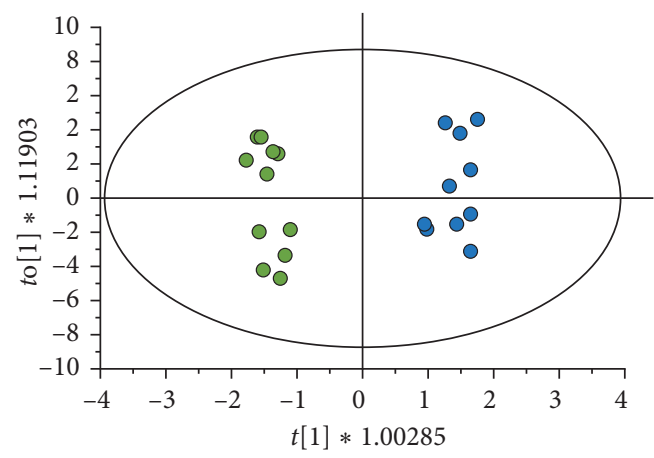

$\square$ DHC1

口 DHC2

(d)

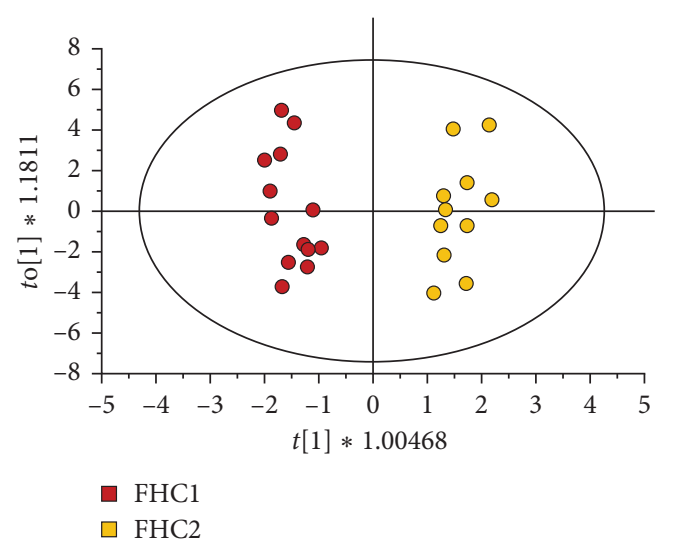

(f)

Figure 4: Continued. 


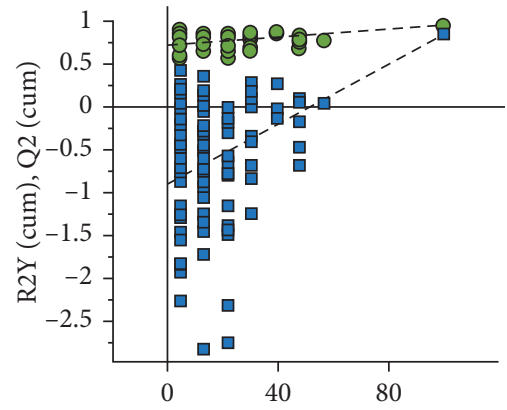

(\%)

O R2Y (cum)

$\square \mathrm{Q} 2(\mathrm{cum})$

(g)

FIGURE 4: PCA analysis and OPLS-DA analysis of DHC and FHC and their two harvest seasons. PCA score plot for DHC and FHC (a). OPLS-DA score plot and the corresponding validation plots based on 200 times permutation tests of the OPLS-DA model for DHC and FHC $(b, c)$, for DHC1 and DHC2 (d, e), and for FHC1 and FHC2 (f, g).

TABle 2: Pharmacological effects of chemical markers identified for DHC and FHC, DHC1 and DHC2, and FHC1 and FHC2 from the OPLS-DA validation plot.

\begin{tabular}{|c|c|c|c|c|c|c|}
\hline & Compound & $\begin{array}{l}\text { Chemical } \\
\text { formula }\end{array}$ & Classification & $p$ value & $\begin{array}{l}\text { VIP } \\
\text { value }\end{array}$ & Pharmacological effects \\
\hline \multirow{11}{*}{$\begin{array}{l}\text { DHC- } \\
\text { FHC }\end{array}$} & $\alpha$-Pinene $^{\mathrm{a}}$ & $\mathrm{C}_{10} \mathrm{H}_{16}$ & $\begin{array}{l}\text { Monoterpene } \\
\text { hydrocarbons }\end{array}$ & $7.37 \times 10^{-8}$ & 1.70 & $\begin{array}{l}\text { Anti-inflammatory [13], antioxidant [14], } \\
\text { antitumor [14], antifungal [14], antiallergy, } \\
\text { improving ulcer [14], antianxiety [14] }\end{array}$ \\
\hline & Limonene $^{\mathrm{b}}$ & $\mathrm{C}_{10} \mathrm{H}_{16}$ & $\begin{array}{l}\text { Monoterpene } \\
\text { hydrocarbons }\end{array}$ & $8.43 \times 10^{-11}$ & 1.76 & $\begin{array}{c}\text { Anti-inflammatory [15], antidiabetic effects } \\
\text { [16], immunomodulatory activity [17], } \\
\text { analgesic [18], hypolipidemic and antioxidant } \\
\text { activities [19], antimicrobial activity [20], } \\
\text { anticancer activity [20], insecticidal activity } \\
\text { [20] }\end{array}$ \\
\hline & $\beta$-Phellandrene ${ }^{c}$ & $\mathrm{C}_{10} \mathrm{H}_{16}$ & $\begin{array}{l}\text { Monoterpene } \\
\text { hydrocarbons }\end{array}$ & $8.75 \times 10^{-7}$ & 2.19 & $\begin{array}{c}\text { Antitumor activity [21], antioxidant activities } \\
\text { [22] }\end{array}$ \\
\hline & $\alpha$-Terpineol $^{\mathrm{d}}$ & $\mathrm{C}_{10} \mathrm{H}_{18} \mathrm{O}$ & $\begin{array}{l}\text { Oxygenated } \\
\text { monoterpenes }\end{array}$ & $5.04 \times 10^{-12}$ & 1.60 & $\begin{array}{c}\text { Anti-inflammatory and analgesic [23], } \\
\text { anticonvulsant [24], gastric protection effects } \\
\text { [25] }\end{array}$ \\
\hline & 4 -Tridecanone ${ }^{e}$ & $\mathrm{C}_{13} \mathrm{H}_{26} \mathrm{O}$ & $\begin{array}{l}\text { Aliphatic } \\
\text { compounds }\end{array}$ & $7.85 \times 10^{-5}$ & 1.53 & - \\
\hline & Ethyl decanoate ${ }^{\mathrm{f}}$ & $\mathrm{C}_{12} \mathrm{H}_{24} \mathrm{O}_{2}$ & $\begin{array}{l}\text { Aliphatic } \\
\text { compounds }\end{array}$ & $1.23 \times 10^{-16}$ & 2.68 & - \\
\hline & Nonanal $^{g}$ & $\mathrm{C}_{9} \mathrm{H}_{18} \mathrm{O}$ & $\begin{array}{l}\text { Aliphatic } \\
\text { compounds }\end{array}$ & $1.03 \times 10^{-7}$ & 1.54 & - \\
\hline & 1-Nonanol ${ }^{\mathrm{h}}$ & $\mathrm{C}_{9} \mathrm{H}_{20} \mathrm{O}$ & $\begin{array}{l}\text { Aliphatic } \\
\text { compounds }\end{array}$ & $8.5810^{-9}$ & 1.68 & - \\
\hline & $\beta$-Cyclocitral ${ }^{\mathrm{i}}$ & $\mathrm{C}_{10} \mathrm{H}_{16} \mathrm{O}$ & $\begin{array}{l}\text { Oxygenated } \\
\text { monoterpenes }\end{array}$ & $2.07 \times 10^{-12}$ & 1.78 & - \\
\hline & $n$-Hexadecanoic acid $^{j}$ & $\mathrm{C}_{16} \mathrm{H}_{32} \mathrm{O}_{2}$ & $\begin{array}{l}\text { Aliphatic } \\
\text { compounds }\end{array}$ & $2.11 \times 10^{-11}$ & 1.67 & Anti-inflammatory [26] \\
\hline & Octadecanol $^{\mathrm{k}}$ & $\mathrm{C}_{18} \mathrm{H}_{38} \mathrm{O}$ & $\begin{array}{l}\text { Aliphatic } \\
\text { compounds }\end{array}$ & $3.58 \times 10^{-8}$ & 1.68 & - \\
\hline \multirow{2}{*}{$\begin{array}{l}\text { DHC1- } \\
\text { DHC2 }\end{array}$} & 4 -Tridecanone $^{1}$ & $\mathrm{C}_{13} \mathrm{H}_{26} \mathrm{O}$ & $\begin{array}{l}\text { Aliphatic } \\
\text { compounds }\end{array}$ & $3.43 \times 10^{-4}$ & 3.34 & - \\
\hline & Ethyl decanoate $^{\mathrm{m}}$ & $\mathrm{C}_{15} \mathrm{H}_{24} \mathrm{O}$ & $\begin{array}{c}\text { Oxygenated } \\
\text { sesquiterpenes }\end{array}$ & $2.39 \times 10^{-3}$ & 2.56 & - \\
\hline
\end{tabular}


TABle 2: Continued.

\begin{tabular}{lcccccc}
\hline Compound & $\begin{array}{c}\text { Chemical } \\
\text { formula }\end{array}$ & Classification & $p$ value & $\begin{array}{c}\text { VIP } \\
\text { value }\end{array}$ & Pharmacological effects \\
\hline$\beta$-Myrcene & $\mathrm{C}_{10} \mathrm{H}_{16}$ & $\begin{array}{c}\text { Monoterpene } \\
\text { hydrocarbons }\end{array}$ & $4.70 \times 10^{-6}$ & 1.67 & $\begin{array}{c}\text { Antioxidant [27], liver monooxygenase } \\
\text { induction effects [28] }\end{array}$ \\
FHC1- & $\beta$-Phellandrene & $\mathrm{C}_{10} \mathrm{H}_{16}$ & $\begin{array}{c}\text { Monoterpene } \\
\text { hydrocarbons }\end{array}$ & $7.60 \times 10^{-3}$ & 2.56 & $\begin{array}{c}\text { Antitumor activity [21], antioxidant activities } \\
\text { [22] }\end{array}$ \\
FHC2 & $\begin{array}{c}\text { 2,6-Octadien-1-ol, 3,7- } \\
\text { dimethyl-, acetate, }(\mathrm{Z})-\end{array}$ & $\mathrm{C}_{12} \mathrm{H}_{20} \mathrm{O}_{2}$ & $\begin{array}{c}\text { Oxygenated } \\
\text { monoterpenes }\end{array}$ & $5.29 \times 10^{-5}$ & 2.15 & - \\
Phytol $^{\mathrm{q}}$ & $\mathrm{C}_{20} \mathrm{H}_{40} \mathrm{O}$ & $\begin{array}{c}\text { Oxygenated } \\
\text { diterpenes }\end{array}$ & $1.27 \times 10^{-3}$ & 2.32 & $\begin{array}{c}\text { Anti-inflammatory [29], antibacterial [30], } \\
\text { antischistosomiasis [31] }\end{array}$ \\
\hline
\end{tabular}

Note: VIP $>1.5$ and $p<0.01$.The symbol “-” stands for none.

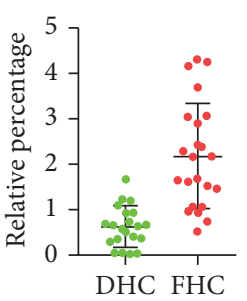

(a)

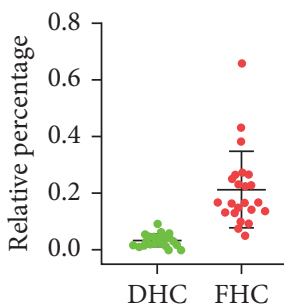

(f)

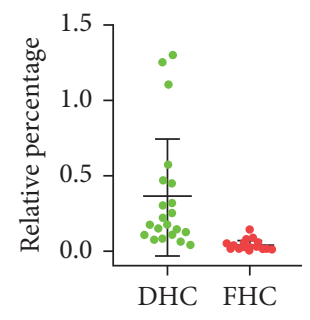

(k)

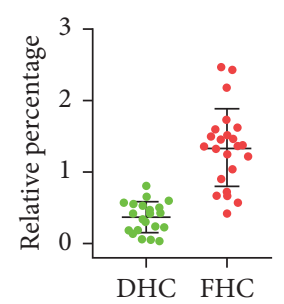

(b)

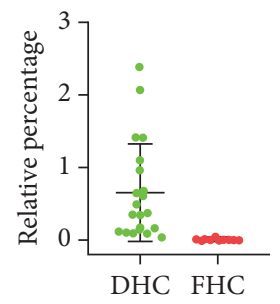

(g)

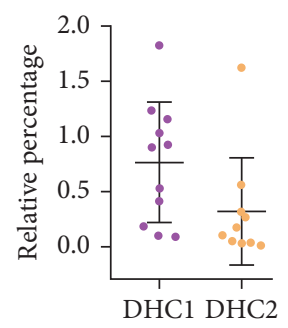

(l)

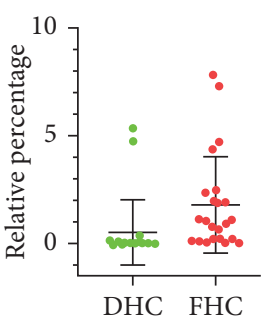

(c)

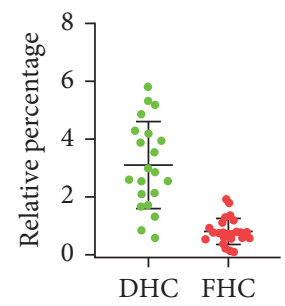

(h)

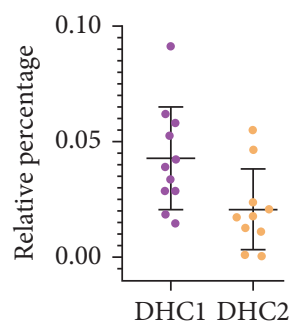

(m)

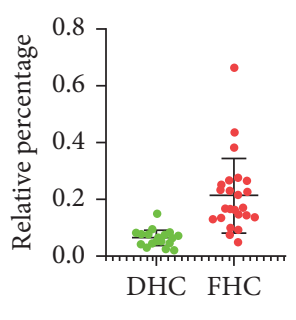

(d)

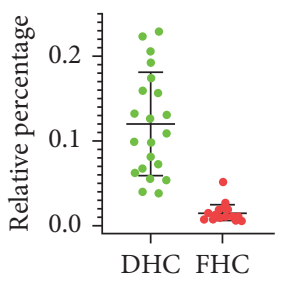

(i)

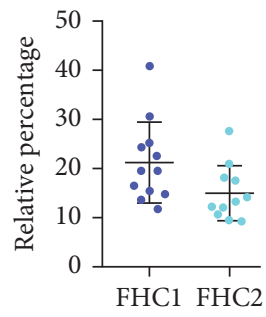

(n)

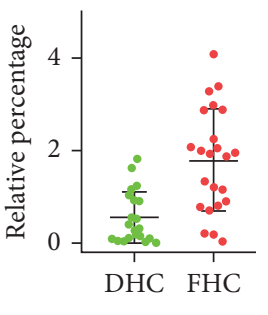

(e)

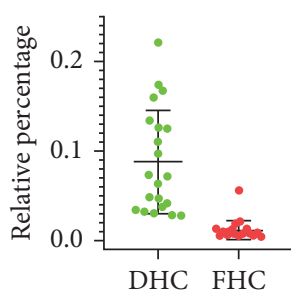

(j)

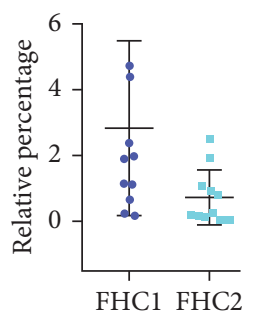

(o)

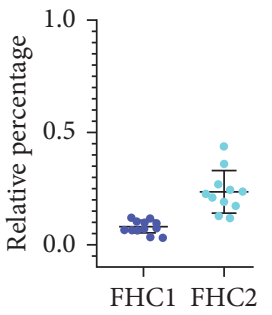

(p)

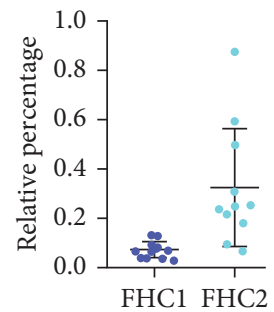

(q)

Figure 5: Relative content of chemical markers of DHC and FHC (a-k), DHC1 and DHC2 (1, m), and FHC1 and FHC2 (n-q). (a) $\alpha$-Pinene; (b) limonene; (c) $\beta$-phellandrene; (d) $\alpha$-terpineol; (e) 4-tridecanone; (f) ethyl decanoate; (g) nonanal; (h) 1 -nonanol; (i) $\beta$-cyclocitral; (j) n-hexadecanoic acid; (k) octadecanol; (l) 4-tridecanone; (m) ethyl decanoate; (n) $\beta$-myrcene; (o) $\beta$-phellandrene; (p) 2,6-octadien-1-ol, 3,7-dimethyl-, acetate, (z)-; (q) phytol. 
were distributed on two sides obviously, as indicated in Figure 4(f). The variation in X (R2Xcum) was 0.637 and the variation in $\mathrm{Y}$ (R2Ycum) was 0.956 , predicting $83.3 \%$ of the variation in response to $\mathrm{Y}(\mathrm{Q} 2 \mathrm{cum}=0.833)$. Four components, including $\beta$-myrcene, $\beta$-phellandrene, 2,6-octadien-1ol,3,7-dimethyl-acetate, and (z)-phytol, were identified as chemical markers for FHC1 and FHC2. All the chemical markers are shown in Table 2.

3.5. Content Comparison of Chemical Markers and Their Pharmacological Effects. Given that the EO of HC exerts various pharmacological activities [2, 3], we explored whether the above chemical markers possess these activities. As present in Table 2, five chemical markers ( $\alpha$-pinene, limonene, $\beta$-phellandrene, $\alpha$-terpineol, and $\mathrm{n}$-hexadecanoic acid) between DHC and FHC and three chemical markers ( $\beta$-myrcene, $\beta$-phellandrene, and phytol) in FHC at two harvest seasons exhibit a wide range of pharmacological activities including anti-inflammatory, ant-oxidant, antiallergy, antibacterial, antiviral, immunologic, anticancer, antianxiety, analgesic, and antidiabetic effects [13-31]. Therefore, the different contents of these chemical markers in DHC and FHC and the two harvest seasons of FHC might influence the quality and efficacy.

As shown in Figure 5, scatter plots were applied to further intuitively compare the content differences of the chemical markers in the three groups (DHC and FHC; DHC1 and DHC2 FHC1 and FHC2). Comparing DHC and FHC, the average contents of $\alpha$-pinene ${ }^{\mathrm{a}}(0.63 \pm 0.44 \%$ and $2.18 \pm 1.15 \%$, respectively), limonene ${ }^{\mathrm{b}}(0.37 \pm 0.22 \%$ and $1.35 \pm 0.55 \%$, respectively), $\beta$-phellandrene ${ }^{c}(0.53 \pm 1.51 \%$ and $1.82 \pm 2.23 \%$, respectively), $\alpha$-terpineol ${ }^{\mathrm{d}}(0.06 \pm 0.03 \%$ and $0.21 \pm 0.13 \%$, respectively), 4 -tridecanone ${ }^{\mathrm{e}}(0.55 \pm 0.56 \%$ and $1.78 \pm 1.11 \%$, respectively), and ethyl decanoate ${ }^{\mathrm{f}}$ $(0.03 \pm 0.02 \%$ and $0.80 \pm 0.46 \%$, respectively) were higher in FHC oils, while the average contents of nonanal ${ }^{\mathrm{g}}$ $\left(0.67 \pm 0.68 \%\right.$ and $0.07 \pm 0.04 \%$, respectively), 1 -nonanol ${ }^{\mathrm{h}}$ $\left(3.10 \pm 1.50 \%\right.$ and $0.49 \pm 0.55 \%$, respectively), $\beta$-cyclocitral ${ }^{\mathrm{i}}$ $(0.12 \pm 0.06 \%$ and $0.01 \pm 0.01 \%$, respectively), n-hexadecanoic acid $(0.09 \pm 0.06 \%$, and $0.01 \pm 0.01 \%$, respectively), and octadecanol ${ }^{\mathrm{k}}(0.36 \pm 0.39 \%$ and $0.04 \pm 0.03 \%$, respectively) from DHC oils were higher than those from FHC oils. Among these markers, $\alpha$-pinene ${ }^{\mathrm{a}}$, limonene ${ }^{\mathrm{b}}$, $\beta$-phellandrene ${ }^{c}$, and $\alpha$-terpineol ${ }^{\mathrm{d}}$ higher in FHC had several pharmacological activities, especially anti-inflammatory $[13,15]$, antioxidant $[14,19]$, antimicrobial $[20]$, and anticancer activities $[14,20,21]$. Meanwhile, only one chemical marker, $n$-hexadecanoic acid', with anti-inflammatory activity [26] was higher in DHC. This result suggested that FHC should be considered as the first choice when it was used as an anti-inflammatory, antioxidant, anticancer, and antibacterial herb. The suggestion was consistent with previous reports [1]. Additionally, the contents of 4-tridecanone $^{1}(0.76 \pm 0.55 \%$ and $0.32 \pm 0.49 \%$, respectively) and ethyl decanoate $\mathrm{e}^{\mathrm{m}}(0.04 \pm 0.02 \%$ and $0.02 \pm 0.02 \%$, respectively) were higher in DHC1 oils than DHC2 oils. The contents of $\beta$-myrcene ${ }^{\mathrm{n}}(21.33 \pm 8.72 \%$ and $15.12 \pm 5.58 \%$, respectively) and $\beta$-phellandrene ${ }^{\circ} \quad(2.82 \pm 2.65 \%$ and
$0.73 \pm 0.83 \%$, respectively) higher in FHC1 oils had antitumor [21] and antioxidant activities [22, 27], while the contents of 2,6-octadien-1-ol, 3,7-dimethyl-, acetate ${ }^{\mathrm{p}}$ $\left(0.08 \pm 0.03 \%\right.$ and $0.23 \pm 0.09 \%$, respectively), and $(\mathrm{z})-$ phytol $^{\mathrm{q}}$ $(0.07 \pm 0.03 \%$ and $0.33 \pm 0.24 \%$, respectively) with anti-inflammatory and antibacterial activities $[29,30]$ were higher in FHC2 oils.

\section{Conclusions}

In this work, GS-MS analysis and chemometric methods were applied to compare the oils of DHC and FHC in two harvest seasons. The results showed that $\mathrm{EO}$ yields of FHC were higher than DHC, and $\mathrm{EO}$ yields of $\mathrm{DHC} 1 / \mathrm{FHC1}$ collected in summer were higher than of DHC2/FHC2 collected in autumn. GC-MS fingerprints showed that DHC and FHC were similar in general and 69 common chemical constituents were characterized. 2-Undecanone, $\beta$-myrcene, and $\beta$-pinene (accounting for about $70 \%$ of the total) were the three most abundant components in both DHC and FHC. Nevertheless, the contents of some constituents were significantly different such as $\alpha$-pinene, $\beta$-phellandrene, 1 nonanol, and geranyl acetate. Moreover, the results of chemometric analysis including HCA, PCA, and OPLS-DA indicated obvious distinction between DHC and FHC and the two harvest seasons of DHC (DHC1 and DHC2) and FHC (FHC1 and FHC2), and OPLS-DA further revealed 11, 2 , and 4 components as their potential chemical markers, respectively. Through this study, we found that the processing methods (i.e., medicinal parts and drying process) and harvest seasons can directly affect the chemical composition of HC and their quality. Future studies are needed to verify whether the differences between them would influence the pharmacological effects to provide a better reference for clinical medication.

\section{Data Availability}

The data used to support the finding of this study are included within the article.

\section{Conflicts of Interest}

The authors declare no conflicts of interest.

\section{Authors' Contributions}

Dingfang Chen, Kaiwen Deng, Lei Meng, and Meifeng Xiao were responsible for data curation; Yalun Zhang.; Methodology, Jinjin Zheng, Ronghua Yin, and Fuyuan He conducted the investigation; Juan Zou acquired the resources; Xue Pan and Haiying Li wrote the original draft. All authors have read and agreed to the published version of the manuscript. Xue Pan and Haiying Li contributed equally to this work.

\section{Acknowledgments}

This work was financially supported by the Pharmaceutical Open Fund of Domestic First-Class Disciplines of Hunan 
Province (2018YX06 and 2020YX13) and Changsha Bureau of Science and Technology (kq1907137).

\section{References}

[1] J. Meng, K. S. Leung, Z. Jiang, X. Dong, Z. Zhao, and L. J. Xu, "Establishment of GC-MS fingerprint of fresh Houttuynia cordata," Chemical \& Pharmaceutical Bulletin, vol. 53, no. 12, pp. 1484-1489, 2005.

[2] O. Se, "An effective quality control of pharmacologically active volatiles of Houttuynia cordata Thunb by fast gas chromatography-surface acoustic wave sensor," Molecules, vol. 20, no. 6, pp. 10298-10312, 2015.

[3] R. S. Verma, N. Joshi, R. C. Padalia et al., "Chemical composition and allelopathic, antibacterial, antifungal, and antiacetylcholinesterase activity of fish-mint (Houttuynia cordata Thunb.) from India," Chemistry \& Biodiversity, vol. 14, Article ID e1700189, 2017.

[4] Y. Asakawa, K. Tomiyama, K. Sakurai, Y. Kawakami, and Y. Yaguchi, "Volatile compounds from the different organs of Houttuynia cordata and litsea cubeba (L. Citriodora)," Journal of Oleo Science, vol. 66, no. 8, pp. 889-895, 2017.

[5] J. Chen, W. Wang, C. Shi, J. Wan, L. Deng, and J. Fang, "Determination of four volatile compounds with anti-inflammatory biological activity in Houttuynia chordata Thunb. by gas chromatography and gas chromatography-mass Spectrometry," Analytical Letters, vol. 47, no. 5, pp. 730-741, 2014.

[6] H. Ao, J. Wang, L. Chen, S. Li, and C. Dai, "Comparison of volatile oil between the fruits of Amomum villosum Lour. and Amomum villosum Lour. var. xanthioides T. L. Wu et Senjen based on GC-MS and chemometric techniques," Molecules (Basel, Switzerland), vol. 24, no. 9, Article ID 1663, 2019.

[7] Y. Hu, W. Kong, X. Yang, L. Xie, J. Wen, and M. Yang, "GCMS combined with chemometric techniques for the quality control and original discrimination of Curcumae longae rhizome: analysis of essential oils," Journal of Separation Science, vol. 37, no. 4, pp. 404-411, 2014.

[8] C. Wang, C. X. Zhang, C. F. Shao et al., "Chemical fingerprint analysis for the quality evaluation of deepure instant $\mathrm{Pu}$-erh tea by HPLC combined with chemometrics," Food Analytical Methods, vol. 9, no. 12, pp. 1-12, 2016.

[9] W. F. Yang, Y. Chen, and J. Y. Cheng, "Study on the volatile components of different parts of Houttuynia cordata," Chinese Traditional and Herbal Drugs, vol. 37, pp. 1149-1151, 2006.

[10] H. Lu, X. Wu, Y. Liang, and J. Zhang, "Variation in chemical composition and antibacterial activities of essential oils from two species of Houttuynia THUNB," Chemical and Pharmaceutical Bulletin, vol. 54, no. 7, pp. 936-940, 2006.

[11] H. M. Lu, Y. Z. Liang, X. J. Wu, L. Z. Yi, and S. Chen, "Comparative study of fingerprints of Hou ttuynia cordata injection made of fresh and dry raw material," Chinese Journal of AnalyticalChemistry, vol. 34, no. 6, pp. 813-816, 2006.

[12] L. H. Lan, A Comparative Study between the Active Components of Fresh and Dry Medicinal Houttuynia Thunb, Zhejiang University of Technology, Hangzhou, China, 2012.

[13] S.-Y. Nam, C.-K. Chung, J.-H. Seo, S.-Y. Rah, H.-M. Kim, and H.-J. Jeong, "The therapeutic efficacy of $\alpha$-pinene in an experimental mouse model of allergic rhinitis," International Immunopharmacology, vol. 23, no. 1, pp. 273-282, 2014.
[14] F. H. Zhu, C. Zhang, and F. X. Wei, "Overview of research on pharmacology and application of alpha pinene," Hans Journal of Medicinal Chemistry, vol. 3, no. 3, pp. 23-28, 2015.

[15] S. Chaudhary, M. Siddiqui, M. Athar, and M. S. Alam, "DLimonene modulates inflammation, oxidative stress and RasERK pathway to inhibit murine skin tumorigenesis," Human \& Experimental Toxicology, vol. 31, no. 8, pp. 798-811, 2012.

[16] M. R. Saravanan, "Antidiabetic effect of d-limonene, a monoterpene in streptozotocin-induced diabetic rats," Biomedicine \& Preventive Nutrition, vol. 2, no. 4, pp. 269-275, 2012.

[17] T. J. Raphael and G. Kuttan, "Immunomodulatory activity of naturally occurring monoterpenes carvone, limonene, and perillic acid," Immunopharmacology and Immunotoxicology, vol. 25, no. 2, pp. 285-294, 2003.

[18] J. F. do Amaral, M. I. G. Silva, M. R. A. de Aquino Neto et al., "Antinociceptive effect of the monoterpene R-(+)-Limonene in mice," Biological and Pharmaceutical Bulletin, vol. 30, no. 7, pp. 1217-1220, 2007.

[19] S. Ahmad and Z. H. Beg, "Hypolipidemic and antioxidant activities of thymoquinone and limonene in atherogenic suspension fed rats," Food Chemistry, vol. 138, no. 2-3, pp. 1116-1124, 2012.

[20] P. Erasto and A. M. Viljoen, "Limonene-a review: biosynthetic, ecological and pharmacological relevance," Natural Product Communications, vol. 3, no. 7, pp. 1193-1202, 2008.

[21] G. Y. Zhang, L. L. Gong, and R. M. Dang, "Analysis on main components and antitumor activities of essential oil from fissistigma cavaleriei leaves," Guizhou Agricultural Sciences, vol. 40, no. 9, pp. 67-69, 2012.

[22] J. J. Xu, J. Q. Lu, X. S. Li et al., "Study on spectrum-effect relationship between fingerprints of essential oil from Shennong Xiangju and the in vitro antioxidant activities," Chinese Journal of Pharmaceutical Analysis, vol. 36, no. 10, pp. 1823-1830, 2016.

[23] D. O. P. D. Sousa, F. Nóbrega, L. Morais, and R. Almeida, "Evaluation of the anticonvulsant activity of terpinen-4-ol," Zeitschrift Fur Naturforschung C, vol. 64, no. 1-2, pp. 1-5, 2009.

[24] J. S. Batista, D. D. Sousa, J. P. Silva et al., "Gastroprotective activity of $\alpha$-terpineol in two experimental models of gastric ulcer in rats," Daru Journal, vol. 19, no. 4, pp. 277-281, 2011.

[25] M. Oliveira, R. B. Marques, M. Santana et al., " $\alpha$-terpineol reduces mechanical hypernociception and inflammatory response," Basic \& Clinical Pharmacology \& Toxicology, vol. 111, no. 2, pp. 120-125, 2012.

[26] V. Aparna, K. V. Dileep, P. K. Mandal, P. Karthe, C. Sadasivan, and M. Haridas, "Anti-inflammatory property of n-hexadecanoic acid: structural evidence and kinetic assessment," Chemical Biology \& Drug Design, vol. 80, no. 3, pp. 434-439, 2012.

[27] O. Ciftci, I. Ozdemir, S. Tanyildizi, S. Yildiz, and H. Oguzturk, "Antioxidative effects of curcumin, $\beta$-myrcene and 1,8-cineole against 2,3,7,8-tetrachlorodibenzo-p-dioxin-induced oxidative stress in rats liver," Toxicology and Industrial Health, vol. 27, no. 5, pp. 447-453, 2011.

[28] A. C. A. X. De-Oliveira, L. F. Ribeiro-Pinto, S. S. Otto, A. Gonçalves, and F. J. R. Paumgartten, "Induction of liver monooxygenases by $\beta$-myrcene,” Toxicology, vol. 124 , no. 2 , pp. 135-140, 1997.

[29] R. O. Silva, F. B. M. Sousa, S. R. B. Damasceno et al., "Phytol, a diterpene alcohol, inhibits the inflammatory response by reducing cytokine production and oxidative stress," 
Fundamental \& Clinical Pharmacology, vol. 28, no. 4, pp. 455-464, 2014.

[30] M. T. Ghaneian, M. H. Ehrampoush, A. Jebali, S. Hekmatimoghaddam, and M. Mahmoudi, "Antimicrobial activity, toxicity and stability of phytol as a novel surface disinfectant," Environmental Health Engineering and Management Journal, vol. 2, no. 1, pp. 13-16, 2015.

[31] J. D. Moraes, R. D. Oliveira, J. P. Costa et al., "Phytol, a diterpene alcohol from chlorophyll, as a drug against neglected tropical disease schistosomiasis mansoni," Plos Neglected Tropical Diseases, vol. 8, no. 1, Article ID e2617, 2014. 